\title{
A segmentation algorithm for noisy images
}

\author{
Soufiane Rital ${ }^{1}$, Hocine Cherifi $^{2}$ and Serge Miguet $^{1}$ \\ 1 LIRIS CNRS, Lyon II University, Lyon, France. \\ \{Soufiane.Rital, Serge.Miguet\}@liris.cnrs.fr, \\ 2 LIRSIA, University of Bourgogne, Dijon, France. \\ Hocine.Cherifi@u-bourgogne.fr
}

\begin{abstract}
This paper presents a segmentation algorithm for gray-level images and addresses issues related to its performance on noisy images. It formulates an image segmentation problem as a partition of a weighted image neighborhood hypergraph. To overcome the computational difficulty of directly solving this problem, a multilevel hypergraph partitioning has been used. To evaluate the algorithm, we have studied how noise affects the performance of the algorithm. The $\alpha$-stable noise is considered and its effects on the algorithm are studied.

Key words : graph, hypergraph, neighborhood hypergraph, multilevel hypergraph partitioning, image segmentation and noise removal.
\end{abstract}

\section{Introduction}

Image segmentation is an important step in computer vision. Several algorithms have been introduced to tackle this problem. Among them are approaches based on graph partitioning [1-3]. Their common point is the building of a weighted graph. This graph is partitioned into components in a way that minimizes a specified cost function of the vertices in the components and/or the boundary between those components. One of the most frequently used techniques to partition a graph is by means of the cut cost function. Several alternatives to the cut criterion have been proposed [1-3]. Of particular note is the normalized cut criterion (Ncut) of Shi and Malik [1], which attempts to rectify the tendency of the cut algorithm to favor isolated nodes of the graph. Also, like graphs, hypergraphs may be partitioned such that a cut metric is minimized. However, hypergraph cut metrics provide a more accurate model than graph partitioning in many cases of practical interest [4]. It has been shown that, in general, there does not exist a graph model that correctly represents the cut properties of the corresponding hypergraph [5]. Recently, several serial and parallel hypergraph partitioning techniques have been extensively studied $[6,7]$ and tools support exists (e.g. hMETIS [8], PaToH [4] and Parkway [9]). These partitioning techniques showed a great efficiency in distributed databases and VLSI circuits fields.

In practice, the images are often corrupted by a noise. Indeed, the noise influences considerably the segmentation quality. To solve this problem, many methods integrate a pre-filtering step. The segmentation quality is conditioned by this step, and more precisely by preserving the useful information. The latter 
one can be assured by using conditional filters. Only the noisy pixels are filtered. Our goal in this paper is to create a hybrid method between a conditional noise removal algorithm and an image segmentation algorithm. The two algorithms use a combinatorial model of the hypergraph theory. The hybrid method integrates three goals: (1) the use of a combinatorial model which adapts perfectly to the image. This model can be used to model a variety of systems, where the relations between objects in a system play a dominant role. (2) The implementation of a multilevel hypergraph partitioning algorithm. (3) The use of a structural noise model. These objectives are gathered in an algorithm which processes in three steps. In the first step, we generate the weighted hypergraph of an image, while in the second step we remove the noise. Only the noisy pixels are filtered. In the last step, we partition the weighted hypergraph into $k$ regions.

The remainder of this paper is organized as follows: in section 2, we introduce the weighted image neighborhood hypergraph. In section 3, we define the structural model of noise. The hypergraph partitioning is introduced in section 4 . In section 5, we illustrate the performance of the proposed segmentation approach. The paper ends with a conclusion in section 6 .

\section{Weighted Image Neighborhood Hypergraph (WINH)}

A hypergraph $H$ on a set $X$ is a family $\left(E_{i}\right)_{i \in I}$ of non-empty subsets of $X$ called hyperedges with : $\cup_{i \in I} E_{i}=X, I=\{1,2, \ldots, n\}, n \in \mathbb{N}$. Given a graph $G(X ; e)$, where $X$ is a set of vertices, and $e$ is a set of unordered pairs of members of $X$ called edges. The hypergraph having the vertices of $G$ as vertices and the neighborhood of these vertices as hyperedges (including these vertices) is called the neighborhood hypergraph of graph $G$. To each $G$ we can associate a neighborhood hypergraph: $H_{G}=\left(X,\left(E_{x}=\{x\} \cup \Gamma(x)\right)\right)$ where $\Gamma(x)=\{y \in$ $X,(x, y) \in e\}$.

Let $H_{G}=\left(X ;\left(E_{i}\right)_{i \in I}\right)$ be a hypergraph. A chain is a sequence of hyperedges $E_{x}$. It is disjoined if the hyperedges $E_{x}$ are not connected two by two. An hyperedge $E_{i}$ is isolated if and only if : $\forall j \in I, j \neq i$ if $E_{i} \cap E_{j} \neq \emptyset$ then $E_{j} \subseteq E_{i}$.

The image will be represented by the following mapping : $I: X \subseteq \mathbb{Z}^{2} \longrightarrow$ $C \subseteq \mathbb{Z}^{n}$. Vertices of $X$ are called pixels, elements of $C$ are called colors. A distance $d$ on $X$ defines a grid (a connected, regular graph, without both loop and multi-edge). Let $d^{\prime}$ be a distance on $C$, we have a neighborhood relation on an image defined by: $\Gamma_{\lambda, \beta}(x)=\left\{x^{\prime} \in X, \mid d^{\prime}\left(I(x), I\left(x^{\prime}\right)\right) \leq \lambda\right.$ and $\left.d\left(x, x^{\prime}\right) \leq \beta\right)$.

The neighborhood of $x$ on the grid will be denoted by $\Gamma_{\lambda, \beta}(x)$. To each image we can associate a hypergraph called Image Neighborhood Hypergraph (INH): $H_{\Gamma_{\lambda, \beta}}=\left(X,\left(\{x\} \cup \Gamma_{\lambda, \beta}(x)\right)_{x \in X}\right)$.

On a grid $\Gamma_{\beta}$, to each pixel $x$ we can associate a neighborhood $\Gamma_{\lambda, \beta}(x)$, according to a predicate $\lambda$. The threshold $\lambda$ can be carried out in two ways. In the first way, the $\lambda$ is given for all the pixels of the image. In the second way, the $\lambda$ is generated locally and applied in an adaptive way to the unit of the pixels.

From $H_{\lambda, \beta}$, we define a Weighted Image Neighborhood Hypergraph (WINH) according to the two maps functions $f_{w_{v}}$ and $f_{w_{h}}$. The first map $f_{w_{v}}$, associates 
an integer weight $w_{x i}$ with every vertex $x_{i} \in X$. The weight is defined by the color in each pixel. The map function $f_{w_{h}}$ associates to each hyperedge a weight $w_{h i}$ defined by the mean color in hyperedge. According to $\lambda$, we generate two representations: WINH and WAINH using respectively global and local thresholds. The last one is named: Weighted Adaptive Image Neighborhood Hypergraph.

\section{Noise Model Definition}

In this section, we define a structural noise model. This model exploits a lack of homogeneity criterion. We consider that the non-homogeneity characterizes noise. The isolated hyperedge can be used to model this non-homogeneity in an image. It is a hyperedge which does not have any information shared with its open neighborhood in the image. We call open neighborhood of the hyperedge $E$ noted $\Gamma^{o}(E)$, the set $\Gamma(E) \backslash E$.

By using this property, we propose the following noise definition: $E_{\lambda, \beta}(x)$ is a noise hyperedge if it verifies one of the two conditions : (1) The cardinality of $E_{\lambda, \beta}(x)$ is equal to 1 and $E_{\lambda, \beta}(x)$ is not contained in disjoined thin chain having $\omega$ elements at least. (2) $E_{\lambda, \beta}(x)$ is an isolated hyperedge and there exists an element $y$ belonging to the open neighborhood of $E_{\lambda, \beta}(x)$ on the grid, such that $E_{\lambda, \beta}(y)$ is isolated.

\section{Multilevel WINH Partitioning}

The formal definition of the $k$-way hypergraph partitioning technique is as follows: find $k$ disjoint subsets $X_{i},(i=0, \ldots, k-1)$ of the vertex set $X$ with part (region) weights $W_{i}(i=0, \ldots, k-1)$ (given by the sum of the constituent vertex weights), such that, given a prescribed balance criterion $0<\epsilon<1$, $W_{i}<(1+\epsilon) W_{\text {avg }}$ holds $\forall i=0, \ldots, k-1$ and an objective function over the hyperedges is minimized. The $W_{\text {avg }}$ denotes the average part weight.

If the objective function is the hyperedge cut metric, then the partition cost (or cut-size) is given by the sum of the costs of hyperedges that span more than one part.

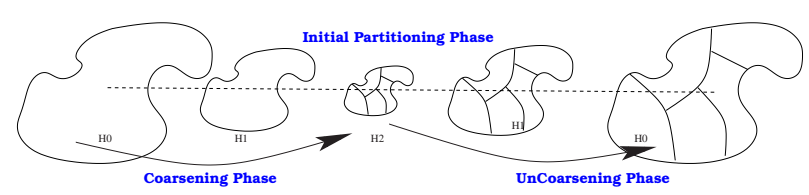

Fig. 1. Multilevel Hypergraph Partitioning.

Computing the optimal bisection or k-section of a hypergraph under the hyperedge cut metric is known to be NP-complete [10]. Thus, researches have 
focused on developing polynomial time heuristic algorithms resulting in good sub-optimal solutions. Because it scales well in terms of run time and solution quality with increasing problem size, the multilevel paradigm is preferred to direct solution approaches. Below, we describe the main steps of the multilevel paradigm (figure. 1):

- Coarsening phase: $H_{\lambda, \beta}$ is approximated via a succession of smaller hypergraphs that maintain its structure as accurately as possible. Many approaches have been proposed for finding the groups of vertices to be merged [7].

- Initial partitioning phase: During the initial partitioning phase, a partitioning of the coarsest hypergraph $H_{\lambda, \beta}{ }^{\text {coarse }}$ is computed, such that it minimizes the cut.

- Uncoarsening phase: During the uncoarsening phase, a partitioning of the coarser hypergraph is successively projected to the next level finer hypergraph, and a partitioning refinement algorithm is used to reduce the cut-set.

Figure 2 illustrates the proposed algorithm. It starts with a WINH generation, noise removal using structural noise model followed by a multilevel hypergraph partitioning.

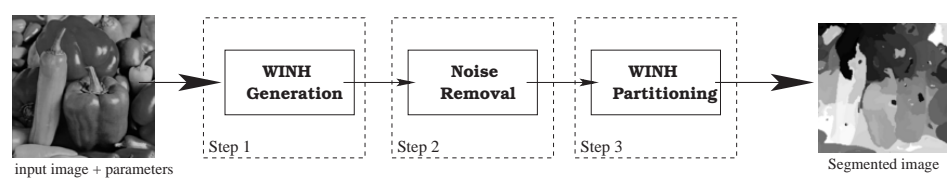

Fig. 2. The three steps of the proposed segmentation algorithm. The input parameters are: $\lambda, \beta, \omega$ and $k$ desired regions.

\section{$5 \quad$ Experimental Results}

We shall present a set of experiments in order to assess the performance of the segmentation approach we have discussed so far. The experimental results contain two steps. In the first step, we evaluate only the segmentation method in non-corrupted images. The algorithm is carried out in two stages: weighted image neighborhood generation followed by a multilevel hypergraph partitioning. In the second step, we evaluate the segmentation method in corrupted images. In this step, the algorithm is carried out in three stages. We start with an evaluation of noise model, then we evaluate the segmentation algorithm in noisy images. For all experiments: In WINH generation, we use the parameters values $\beta, \lambda$ and $k$ adjusted in experiments. In the case of WAINH generation, we use an adaptive threshold $\lambda$ estimated using: $\lambda=\operatorname{Median}\{I(y)-\operatorname{Median}(F(x))\}_{\forall y \in F} . F$ is the window centered in $x$ with the size $[2 \beta+1 \times 2 \beta+1]$. In WINH partitioning, 
and in the coarsening phase, we use the hyperedge coarsening approach (figure 3). In the initial partitioning phase, we compute the k-way partitioning of the coarsest hypergraph using the multilevel hypergraph bisection algorithm [7]. In the uncoarsening phase, we use the F.M. refinement algorithm [6].

For the coarsening, initial partitioning and uncoarsening phases we use the Hmetis package [8].

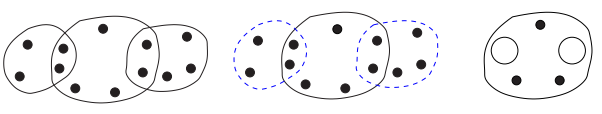

Fig. 3. Hyperedge coarsening method

We will now show the effect of the weighted hypergraph generation on the quality of the image segmentation results. For this study, we implement two weighted neighborhood hypergraph representation : WINH and WAINH. Figure 4 shows the segmentation results of Peppers image. From this figure, we can see that using the WAINH, we obtain significant and better results. Indeed, using WAINH, we detect more significant regions compared to segmentation approach using WINH representation.
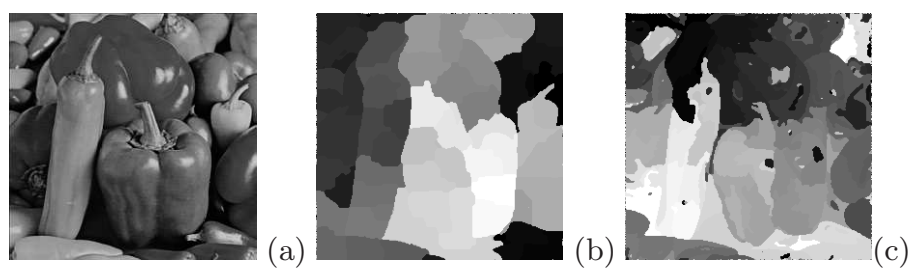

Fig. 4. WINH and WAINH comparison. (a) the original image of size $256 \times 256$. Outputs of our algorithm : (b) using WINH with $(\lambda, \beta, k)=(10,1,51)$ and (c) using WAINH with $(\beta, k)=(1,51)$.

In order to compare our method with an existing one, we have chosen the technique of Shi and Malik (Normalized Cuts detection - Ncut) [1]. We have processed a group of images with our segmentation method and compared the results to Ncut algorithm. The Ncut algorithm used the same parameters for all images, namely, the optimal parameters given by authors. Figure 5 shows a comparison between the proposed and Ncut algorithms on Peppers and Medical images. According to the segmentation results on these images, we note that our algorithm make a better localization of the regions in the processed image compared to the Ncut method. The strength of this algorithm is that it better detects the regions containing many details. In addition, it results in shorter 
computing times faster than Ncut algorithm. The computing times of these two algorithms have been implemented using $\mathrm{C}++$ language in a notebook with the following characteristics: Pentium Centrino, 1.5GHz, 512 Mo RAM.
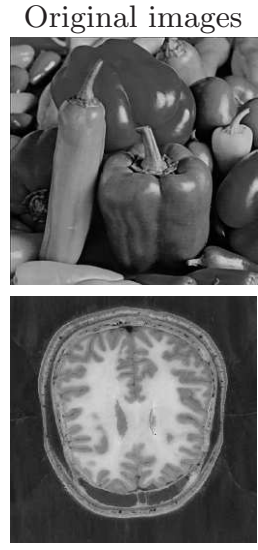

(a)
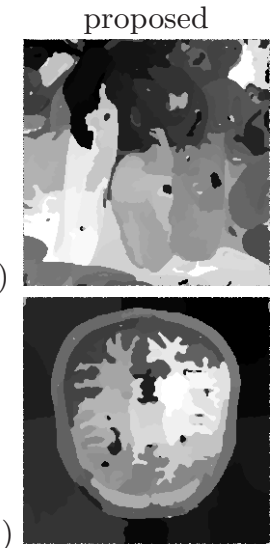

(b)

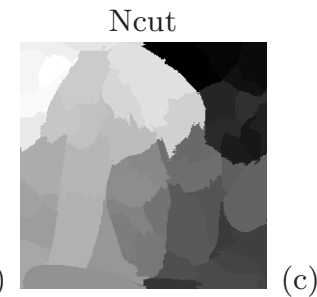

b')

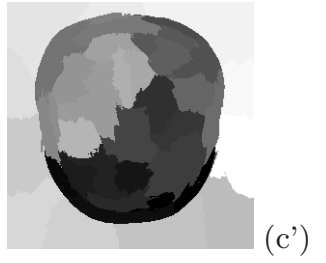

Fig. 5. A comparison between the proposed and Ncut algorithms. (b,b') The outputs processed in $32,23 \mathrm{~s}$ and $29,06 \mathrm{~s}$ respectively. (c,c') The outputs processed in 402,75s with $k=51$ and $463,64 \mathrm{~s} k=40$ respectively. The parameters of $\left(\mathrm{b}^{\mathrm{b}} \mathrm{b}^{\prime}\right)$ are $((\beta, k)=(1,51))$ and $((\beta, k)=(1,39))$ respectively.

Using the noise model illustrated in section 3, we develop a conditional noise removal algorithm. It is conditional because only the noisy hyperedges are filtered. The noise removal algorithm starts with AINH representation followed by noisy hyperedge detection and followed by noisy hyperedge estimation. We tested the performance of noise removal algorithm in the presence of $\alpha$-stable noise. This distribution is a useful model of noise distribution. For a symmetrical distribution, the characteristic function is given by: $\varphi(t)=e^{\left\{j a t-\gamma|t|^{\alpha}\right\}}$, where: (1) $\alpha$ is the characteristic exponent satisfying $0<\alpha \leq 2$. The characteristic exponent controls the heaviness of the tails of the density function. The tails are heavier, and thus the noise more impulsive, for low values of $\alpha$ while for a larger $\alpha$ the distribution has a less impulsive behavior. (2) $a$ is the location parameter $(-\infty<a<+\infty)$. (3) $\gamma$ is the dispersion parameter $(\gamma>0)$, which determines the spread of the density around its location parameter.

The objective of the filtering is to remove the noisy hyperedges while preserving the noise-free patterns. In figure 6 , we present the results of the noise detection in Peppers and Medical images corrupted by $\alpha$-stable noise with two parameters: $\alpha=0.5$ and $\alpha=1,5$ representing respectively a impulsive and Gaussian distribution noise. These two results are compared with the Median Filter. It operates using $3 \times 3$ square processing windows. From the error images $6(\mathrm{e}, \mathrm{e})$ between the filtered image and the original image, we note that the 
proposed algorithm preserves better the edge of the corrupted image than the Median filter.
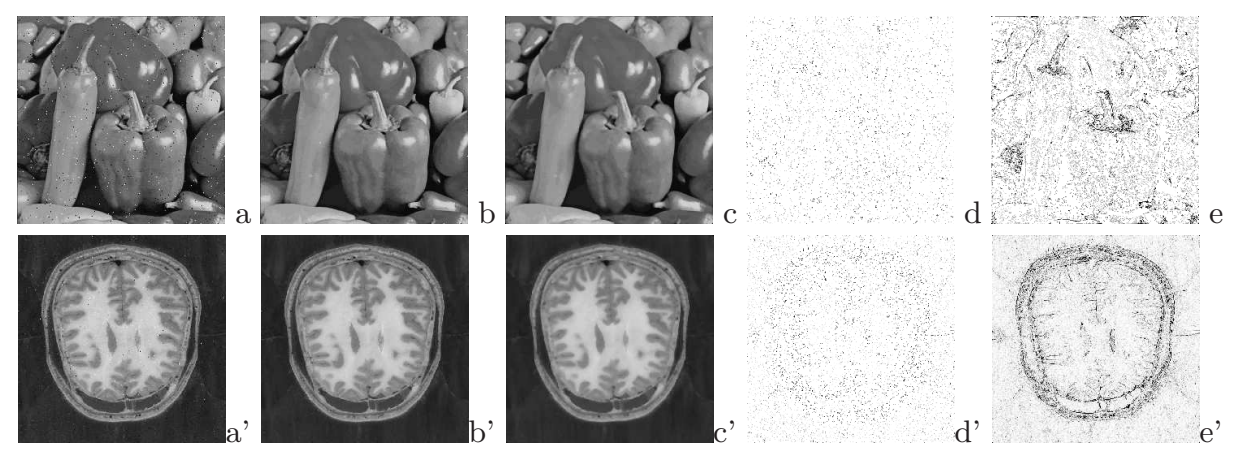

Fig. 6. Noise model evaluation. (a,a') corrupted images by $\alpha$-stable noise. The $\alpha$-stable noise parameters are $(\alpha=0.5, \gamma=1, a=0$, percentage $=10 \%)$ and $(\alpha=1,5, \gamma=20$, $a=0$ and percentage $=10 \%$ ) for images a, a' respectively. (b,b') filtered image by our noise model $(\beta=1, \omega=5)$. (c, c') output of median $3 \times 3$ filter. $\left(\mathrm{d}, \mathrm{d}^{\prime}\right)$ error $\times 10$ between the orginal images and b,b' images respectively. (e,e') error $\times 10$ between the original images and c,c' images respectively.

Segmentation results of Peppers and Medical images corrupted by the same parameters of $\alpha$-stable noise are illustrated in figure 7. This figure shows the segmentation results with and without the integration of the noise model in the proposed algorithm. In the case of non-integration of noise model in the proposed algorithm, we note that this last one detects the noise like regions (figures $\left.7\left(\mathrm{c}, \mathrm{c}^{\prime}\right)\right)$. This noise influences the segmentation result considerably. The figures 7 (b,b') justify the absence of this drawback in the case of use of noise model. According to this figure and to several simulations on several image types we note that the use of both noise model and hypergraph partitioning in WAINH representation constitutes a robust segmentation algorithm to the noise effect.

\section{Conclusions}

We have presented a segmentation algorithm for noisy images. The segmentation is accomplished in three steps. In the first step, a weighted adaptive image neighborhood hypergraph is generated. In the second stage, a conditional noisy hyperedge removal algorithm is computed. In the last stage, a hypergraph partitioning method is computed using a multilevel technique . Experimental results demonstrate that our approach performs better than Ncut algorithm. It can be improved in several ways (parameters: the function maps, the colorimetric threshold, the unsupervised region number, etc.). 

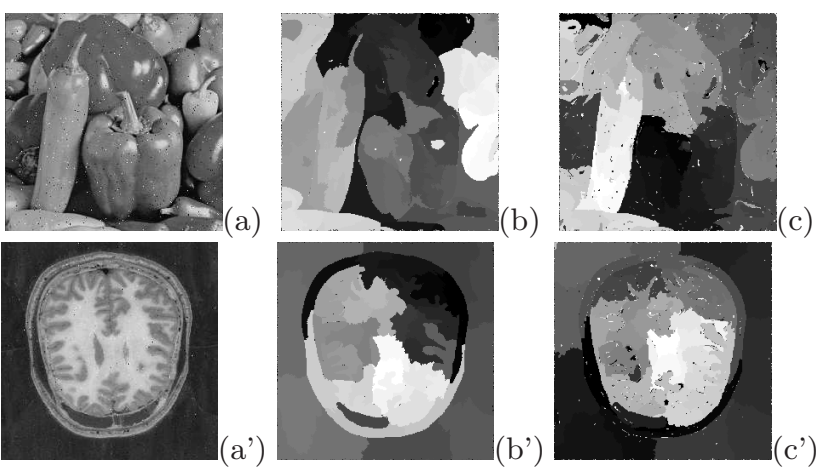

Fig. 7. Robustness evaluation of the proposed algorithm to noise effect. (a,a') corrupted images with $\alpha=0.5, \gamma=1$ and $\alpha=1.5, \gamma=20$ respectively with $10 \%$ of $\alpha$-stable noise. (b,b') The output of the proposed algorithm after noise removal with proposed noise model. (c,c') the output of the proposed algorithm without noise removal. The Peppers image was processed by $\beta=2$ and $k=56$ parameters, while the Medical image used $\beta=2$ and $k=37$ parameters.

\section{References}

1. Shi, J., Malik, J.: Normalized cuts and image segmentation. IEEE Transactions on Pattern Analysis and Machine Intellignece 22 (2000)

2. Martinez, A.M., Mittrapiyanuruk, P., Kak, A.C.: On combining graph-partitioning with non-parametric clustering for image segmentation. Computer Vision and Image Understanding 95 (2004) 72-85

3. Wang, S., Siskind, J.M.: Image segmentation with ratio cut - supplemental material. IEEE Trans. Pattern Anal. Mach. Intell. 25 (2003)

4. Catalyurek, U., Aykanat, C.: Hypergraph-partitioning-based decomposition for parallel sparse-matrix vector multiplication. IEEE Trans. Parallel Distrib. Syst. 10 (1999) 673-693

5. Ihler, E., Wagner, D., Wagner, F.: Modeling hypergraphs by graphs with the same mincut properties. Inf. Process. Lett. 45 (1993) 171-175

6. Sanchis, L.A.: Multiple-way network partitioning. IEEE Transactions on Computers (1989) 6281

7. Karypis, G., Aggarwal, R., Kumar, V., Shekhar, S.: Multilevel hypergraph partitioning: applications in vlsi domain. IEEE Trans. Very Large Scale Integr. Syst. 7 (1999) 69-79

8. Karypis, G., Kumar, V.: hmetis 1.5: A hypergraph partitioning package. Technical report, University of Minnesota, Available on http://www.cs.umn.edu/hmetis (1998)

9. Trifunovic, A., Knottenbelt, W.: Parkway 2.0: A parallel multilevel hypergraph partitioning tool. In: Proceedings of 19th International Symposium on Computer and Information Sciences (ISCIS 2004). Volume 3280. (2004) 789-800

10. Garey, M., Johnson, D.: Computers and Intractability: A Guide to the Theory of NP-Completeness. W.H. Freeman and Co. (1979) 